\title{
Elaboração e aceitabilidade de biscoitos enriquecidos com aveia e farinha de bagaço de uva
}

\section{Elaboration and acceptability of cookies enhanced with oat and flour grape pomace}

\section{Autores | Authors}

\section{Alessandra PIOVESANA} Micheli Maria BUENO

Instituto Federal de Educação, Ciência e Tecnologia do Rio Grande do Sul (IFRS) Departamento de Tecnologia em Alimentos Bento Gonçalves/RS - Brasil e-mail: ale_piovesana@hotmail.com micheli.bueno2008@hotmail.com

\ Vera Maria KLAJN

Instituto Federal de Educação, Ciência e Tecnologia do Rio Grande do Sul (IFRS)

Departamento de Química e

Tecnologia em Alimentos

Av. Osvaldo Aranha, 540.

Bairro Juventude da Enologia CEP: 95700-000

Bento Gonçalves/RS - Brasil e-mail:veraklajn@gmail.com

Autor Correspondente / Corresponding Author

Recebido / Received: 23/05/2012 Aprovado / Approved: 04/02/2013

Publicado / Published: mar./2013

\section{Resumo}

A crescente demanda por alimentos benéficos à saúde é acompanhada pela busca por processos que gerem baixo volume de resíduos sólidos ou que proporcionem seu reaproveitamento. Este trabalho propõe a elaboração e a avaliação da aceitabilidade de biscoitos enriquecidos com aveia e farinha de bagaço de uva. A farinha de bagaço de uva, produzida como alternativa de utilização do grande volume deste resíduo gerado pela indústria vitivinícola, representa uma ótima fonte de fibras e antioxidantes naturais de baixo custo. Esta foi obtida por meio da moagem do bagaço seco e posterior padronização granulométrica. A farinha integral de aveia, fonte de fibras alimentares, foi obtida pela moagem da aveia em flocos. Para a elaboração dos biscoitos, foram utilizadas três formulações com diferentes percentuais de substituição da farinha de trigo por farinha integral de aveia e farinha de bagaço de uva, as quais foram denominadas formulações A, B e C, com níveis de substituição de 30\%, 40\% e $50 \%$, respectivamente. O experimento foi realizado em delineamento inteiramente casualizado, os resultados submetidos à análise de variância e as médias comparadas pelo teste de Tukey a 5\% de significância. Os resultados obtidos demonstraram que os biscoitos elaborados apresentaram propriedades sensoriais aceitáveis, sendo os percentuais de substituição utilizados neste estudo aceitos pelos julgadores, dentre todos os atributos avaliados. O uso da aveia fornece um incremento de fibra alimentar e a farinha de bagaço de uva é um ingrediente alternativo para o reaproveitamento de um produto normalmente descartado.

Palavras-chave: Vitis vinifera; Avena sativa; farinhas mistas; resíduo.

\section{Summary}

The growing demand for healthy food is accompanied by a search for processes that generate low volumes of solid waste, or which provide for its reuse. This paper proposes the development and evaluation of the acceptability of cookies enriched with oat and grape pomace flour. The grape pomace flour, produced as an alternative to the use of large volumes of waste generated by the wine industry, represents a great source of fibre and natural antioxidants at low cost. It was obtained by grinding the dry pomace and subsequent granulometric standardization. The oat flour, a source of food fibres, was obtained by grinding oat flakes. To prepare the cookies, three formulations with different percentages of replacement of the wheat flour by wholemeal oat flour and grape pomace flour were used, defined as Formulations A, B and C with $30 \%, 40 \%$ and $50 \%$ replacement respectively. The experiment was carried out using a completely randomized design, the results submitted to an analysis of variance and the means compared by the Tukey test at $5 \%$ significance. The results demonstrated that the cookies presented acceptable sensory properties and the percentage of substitution used in this study was accepted by the judges for all the attributes evaluated. The use of oat provided an increase in food fibre and the grape pomace flour was an alternative ingredient for the reuse of a product normally discarded.

Key words: Vitis vinifera; Avena sativa; mixed flour; waste. 


\section{Introdução}

A crescente demanda por alimentos seguros e que tragam algum benefício à saúde vem sendo fortemente acompanhada pela busca por processos limpos de produção, uma vez que o tratamento dos resíduos sólidos e líquidos que são gerados acarreta custos cada vez maiores para a indústria de alimentos. Esse é o caso da indústria vinícola, que responde por um volume substancial de resíduos orgânicos sólidos. Como subproduto, o bagaço representa aproximadamente $20 \%$ das uvas colhidas (ROCKENBACH, 2008). As uvas e seus subprodutos não são considerados particularmente nutritivos, uma vez que seu teor vitamínico não é significativo. Entretanto, seu teor de antioxidantes naturais e fibras representam uma propriedade benéfica, fato esse que poderá aumentar seu valor de mercado num futuro próximo (LLOBERA e CAÑELLAS, 2007).

A aveia foi reconhecida como alimento funcional em 1997 pela FDA (Food and Drug Administration) e tem recebido destaque por ser um cereal promotor de saúde em virtude das suas propriedades nutricionais e funcionais, do seu teor e da sua qualidade de fibras alimentares, além de ser uma fonte natural de antioxidantes. Por isso, tem crescido o interesse dos consumidores por produtos que contenham este grão em sua formulação.

Uma alternativa que vem crescendo desde o início da década de 1970 consiste no aproveitamento de resíduos (principalmente cascas) de certas frutas como matéria-prima para a produção de alguns alimentos perfeitamente passíveis de serem incluídos na alimentação humana (ISHIMOTO et al., 2007).

A utilização de farinhas mistas expandiu-se, sendo utilizada na fabricação de biscoitos, já que este é um produto altamente aceito e consumido por pessoas de todas as faixas etárias. Tais características, aliadas à sua enorme diversidade, apresentam-se como uma nova opção para o estudo de diferentes tipos de farinhas e suas propriedades físicas, químicas e sensoriais, possibilitando o aumento das propriedades tecnológicas e funcionais (SILVA et al., 2001; KOPPER et al., 2009).

Este trabalho teve como objetivo propor a elaboração de biscoitos com substituições parciais da farinha de trigo por farinha integral de aveia e farinha de bagaço de uva, bem como verificar a aceitabilidade sensorial e a intenção de compra.

\section{Material e métodos}

Foram utilizadas, como matérias-primas, farinha integral de aveia produzida na indústria de processamento de aveia Saboreal Cereais e Alimentos Ltda, de Ajuricaba-RS e farinha de bagaço de uva (Vitis vinifera), preparada na Agroindústria do Instituto Federal de
Educação, Ciência e Tecnologia do Rio Grande do Sul (IFRS) - Campus Bento Gonçalves. O bagaço, constituído por cascas e sementes de uva, foi previamente seco em estufa com circulação e renovação de ar Solab, modelo SL 102 a $45^{\circ} \mathrm{C}$, até atingir a umidade indicada para a produção de farinhas ( $14 \%$ p/p no máximo), e padronizado granulometricamente por meio do processo de moagem, com moinho rotor Marconi, modelo MA 340. Os demais ingredientes utilizados para a elaboração dos biscoitos foram adquiridos no comércio local.

Os biscoitos foram elaborados seguindo a formulação da Tabela 1. As formulações com diferentes percentuais de substituição da farinha de trigo foram denominadas A, B e C. Para o preparo dos biscoitos, inicialmente, as farinhas foram misturadas (mix) e homogeneizadas nas proporções descritas na Tabela 2.

Para o preparo da massa, inicialmente foram batidos os ovos, o açúcar e a margarina, em batedeira Britânia, modelo turbo 360, por 5 minutos ou até se obter um creme homogêneo (Figura 1). Ao creme, adicionaram-se, aos poucos, os mix de farinha e o fermento, até a

Tabela 1. Ingredientes e quantidades utilizadas na elaboração dos biscoitos.

\begin{tabular}{lc}
\multicolumn{1}{c}{ Ingrediente } & Quantidade (g) \\
\hline Mix de farinhas & 500 \\
Amido de milho & 150 \\
Fermento químico & 15 \\
Açúcar mascavo & 200 \\
Açúcar cristal & 150 \\
Ovos & 150 \\
Margarina com 80\% de lipídios & 150 \\
\hline
\end{tabular}

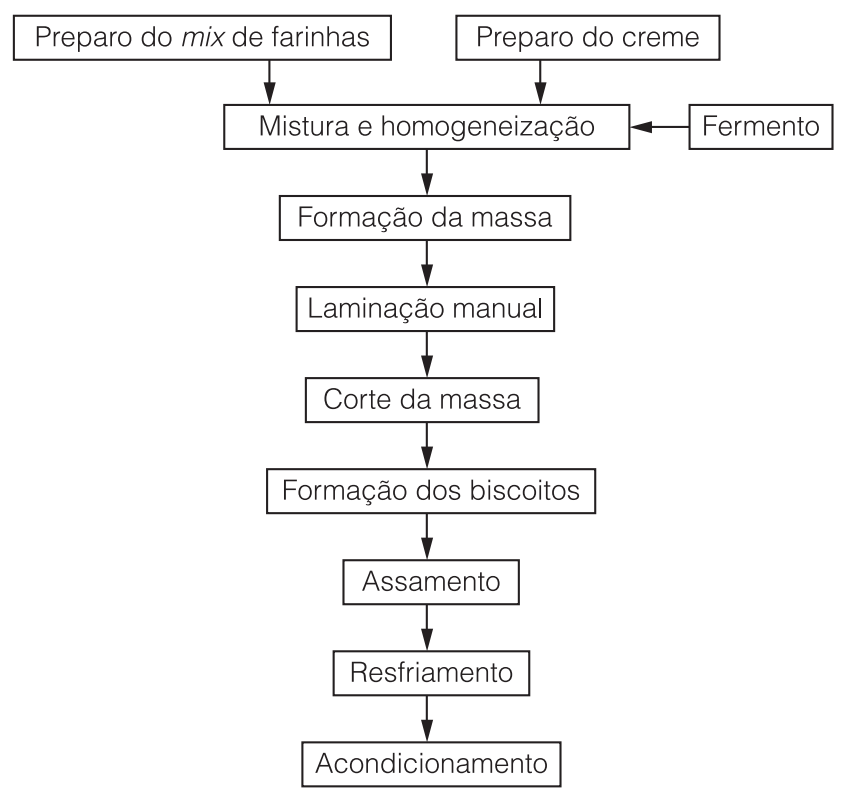

Figura 1. Fluxograma das etapas da elaboração dos biscoitos enriquecidos com aveia e farinha de bagaço de uva. 
Elaboração e aceitabilidade de biscoitos enriquecidos com aveia e farinha de bagaço de uva

PIOVESANA, A. et al.

Tabela 2. Percentuais de farinhas de trigo, integral de aveia e bagaço de uva.

\begin{tabular}{cccc}
\hline Formulações & Farinha de trigo (\%) & Farinha integral de aveia (\%) & Farinha de bagaço de uva (\%) \\
A & 70 & 15 & 15 \\
B & 60 & 20 & 20 \\
C & 50 & 25 & 25 \\
\hline
\end{tabular}

homogeneidade da massa. Posteriormente, os biscoitos foram moldados e padronizados - em torno de $5 \mathrm{~cm}$ de diâmetro e 0,5 cm de espessura - e assados em forno elétrico ITC, modelo Du Chef Plus à temperatura de 180 ${ }^{\circ} \mathrm{C}$ por, aproximadamente, 17 minutos.

As três formulações foram avaliadas quanto à aceitação global e à intenção de compra, em laboratório de avaliação sensorial, por 80 julgadores não treinados, consumidores de biscoitos, com idades entre 15 e 60 anos (de ambos os gêneros), por meio do teste afetivo de aceitabilidade, de acordo com ABNT (1993, 1998). As amostras foram codificadas com três algarismos e dispostas em ordens diferentes a cada julgador, tendo sido utilizada escala hedônica de 9 pontos (1 - detestei a 9 - adorei), avaliando-se os atributos sabor, crocância e avaliação global. Para o teste de intenção de compra, foi utilizada escala de 5 pontos ancorada em seus extremos, com os termos: 1 - certamente não compraria a 5 - certamente compraria.

O experimento seguiu um delineamento inteiramente casualizado. Os resultados obtidos na aceitabilidade sensorial foram analisados estatisticamente por Análise de Variância (ANOVA) e foi aplicado o teste Tukey no nível de 5\% de significância, para comparação entre as médias.

\section{Resultados e discussão}

Os resultados apresentados na Tabela 3 indicam que as formulações de biscoitos estudadas não diferiram significativamente entre si $(p>0,05)$ em relação aos atributos crocância e impressão global, apesar de estas terem apresentado características próprias. Um exemplo de uma característica específica é a coloração mais escura (Figura 2), em função da adição da farinha de bagaço de uva, que apresenta uma concentração expressiva de pigmentos antociânicos. Estes, além de serem potentes antioxidantes, indicam que esta farinha pode ser utilizada como matéria-prima alternativa para a elaboração de biscoitos, pois apresenta crocância e impressão global aceitáveis. Romero et al. (2004) também observaram coloração escura mais intensa em biscoitos formulados com percentuais mais elevados de bagaço de uva.

Os escores médios obtidos no teste de aceitabilidade sensorial das três formulações de biscoitos demonstram que a formulação "C", com maior

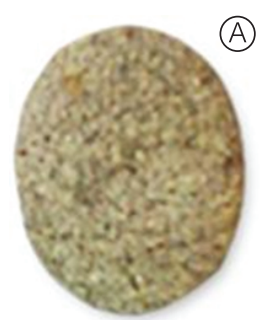

$70: 15: 15$

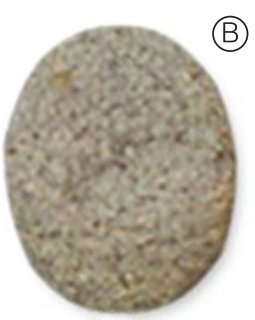

$60: 20: 20$

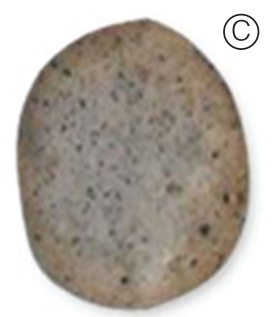

$50: 25: 25$
Figura 2. Biscoitos enriquecidos com aveia e farinha de bagaço de uva. Proporção de farinhas trigo: aveia: bagaço de uva.

Tabela 3. Escore das formulações de diferentes formulações de biscoitos com percentuais de farinhas de trigo, aveia e bagaço de uva em A (70:15:15), B (60:20:20) e C (50:25:25), em relação aos atributos sensoriais sabor, crocância e impressão global.

\begin{tabular}{|c|c|c|c|}
\hline \multirow{2}{*}{ Formulação } & \multicolumn{3}{|c|}{ Atributo Sensorial } \\
\hline & Sabor & Crocância & Impressão global \\
\hline A & $7,23 \pm 1,32^{a}$ & $7,56 \pm 1,13^{a}$ & $7,30 \pm 1,05^{a}$ \\
\hline B & $6,73 \pm 1,40^{\mathrm{ab}}$ & $7,55 \pm 1,05^{a}$ & $6,94 \pm 1,22^{a}$ \\
\hline C & $6,53 \pm 1,40^{b}$ & $7,54 \pm 1,08^{a}$ & $6,86 \pm 1,30^{a}$ \\
\hline
\end{tabular}

Resultados expressos como média \pm desvio padrão; escala hedônica de 9 pontos ( 1 - detestei a 9 - adorei); médias seguidas de mesmas letras na coluna não diferem estatisticamente pelo teste de Tukey a $5 \%$ de probabilidade.

percentual de substituição da farinha de trigo por farinha integral de aveia e farinha de bagaço de uva (25:25) foi a menos aceita quanto ao atributo sabor, diferindo significativamente da formulação " $A$ ", que obteve os melhores resultados. A formulação "B" não difereriu significativamente das demais.

Valores inferiores aos do presente trabalho foram encontrados por Kopper et al. (2009), na análise sensorial empregando farinha de bocaiuva na elaboração de biscoitos tipo cookie, que encontraram valores de 6,14 a 6,58 de aceitação.

OSUNA et al. (2004) avaliaram sensorialmente biscoitos elaborados com variações de bagaço de uva entre 0 e 6,33\%, usando 40 julgadores não treinados e escala hedônica de 9 pontos, e não observaram diferenças significativas, concluindo que há a possibilidade de utilização do bagaço de uva para a elaboração de biscoitos com aceitáveis características sensoriais. Em outro estudo, Romero et al. (2004) elaboraram biscoitos com adição de bagaço de uva em até 10\% e também concluíram que é possível a adição de bagaço de uva 
Elaboração e aceitabilidade de biscoitos enriquecidos com aveia e farinha de bagaço de uva

PIOVESANA, A. et al.

como ingrediente para a fabricação de biscoitos tipo cookies com alto teor de fibras e atributos sensoriais aceitáveis.

Santos et al. (2011) avaliaram sensorialmente biscoitos elaborados com farinha de buriti com e sem adição de aveia, e verificaram boa aceitação em relação aos atributos de sabor, textura, aroma e impressão global, sendo que os biscoitos com adição de aveia obtiveram valores maiores em todas as características citadas em comparação aos sem aveia.

Na análise sensorial de biscoitos de chocolate a partir da incorporação de fécula de mandioca e albedo de laranja, Santos et al. (2010) obtiveram médias entre 6,30 e 6,70 para o atributo impressão global, valores estes inferiores aos encontrados no presente trabalho.

De acordo com Fasolin et al. (2007), outros trabalhos realizados com diferentes tipos de biscoitos têm demonstrado forte tendência das indústrias e de trabalhos de pesquisa em promover o enriquecimento de biscoitos. Segundo Santucci et al. (2003), a mistura de farinhas não convencionais com a farinha de trigo melhora a qualidade nutricional de biscoitos e pode até melhorar sua palatabilidade, tornando-os mais aceitos pelos consumidores.

Para o teste de intenção de compra, os resultados obtidos não diferiram significativamente pelos julgadores, conforme pode ser observado na Tabela 4.

Conforme os escores demonstrados na Tabela 4, os biscoitos apresentaram aceitável intenção de compra pelos julgadores, pois suas notas ficaram entre 3,60 e 3,90, representando as escalas 'talvez comprasse/ talvez não comprasse' e 'possivelmente compraria', respectivamente.

De acordo com os resultados, pode-se afirmar que é possível realizar a substituição da farinha de trigo pelas farinhas integral de aveia e de bagaço de uva na elaboração de biscoitos com aceitáveis características sensoriais e intenção de compra. Estes resultados demonstraram o potencial de aproveitamento do bagaço de uva para o desenvolvimento de biscoitos a partir da farinha obtida deste, constituindo uma nova proposta para seu reaproveitamento, tanto em termos econômicos quanto sustentáveis, pois as grandes quantidades de resíduos gerados pelas vinícolas apresentam sérios problemas de armazenagem ou eliminação.

Tabela 4. Intenção de compra para diferentes formulações de biscoitos com percentuais de farinhas de trigo, aveia e bagaço de uva em A (70:15:15), B (60:20:20) e C (50:25:25).

$\begin{array}{ccccc}\text { Formulação } & \text { A } & \text { B } & \text { C } \\ \text { Intenção de compra } & 3,95 \pm 0,83^{\mathrm{a}} & 3,70 \pm 1,08^{\mathrm{a}} & 3,64 \pm 0,94^{\mathrm{a}}\end{array}$

Resultados expressos como média \pm desvio padrão; escala de 5 pontos ( 1 - certamente não compraria a 5 - certamente compraria); médias seguidas de mesmas letras não diferem estatisticamente pelo teste de Tukey a $5 \%$ de probabilidade.

\section{Conclusões}

Este estudo demonstrou que os biscoitos elaborados com substituição de até $50 \%$ da farinha de trigo por farinha integral de aveia e farinha de bagaço de uva obtiveram uma boa aceitabilidade em relação aos atributos sensoriais avaliados, demonstrando, assim, que é possível utilizar matérias-primas de baixo valor agregado, disponibilizando nova opção de produto saudável.

Os produtos desenvolvidos apresentaram propriedades sensoriais aceitáveis e demonstraram que os níveis de substituição usados neste estudo foram aceitos pelos julgadores, sendo um produto com aceitação comercial satisfatória.

\section{Referências}

ASSOCIAÇÃO BRASILEIRA DE NORMAS TÉCNICAS - ABNT. NBR 12994: Métodos de Análise Sensorial dos Alimentos e Bebidas. Rio de Janeiro: ABNT, 1993. 2 p.

ASSOCIAÇÃO BRASILEIRA DE NORMAS TÉCNICAS - ABNT. NBR 14141: Escalas Utilizadas em Análise Sensorial de Alimentos e Bebidas. Rio de Janeiro: ABNT, 1998. 3 p.

FASOLIN, L. H.; ALMEIDA, G. C.; CASTANHO, P. S.; NETTOOLIVEIRA, E. R. Biscoitos produzidos com farinha de banana: avaliações química, física e sensorial. Ciência e Tecnologia de Alimentos, Campinas, v. 27, n. 3, p. 524-529, 2007. http:// dx.doi.org/10.1590/S0101-20612007000300016

FOOD AND DRUG ADMINISTRATION - FDA. Final rule for food labelling: Health claims; Oats and coronary heart disease. Federal Regulations, Silver Spring, v. 62, p. 3584-3681, 1997.

ISHIMOTO, F. Y.; HARADA, A. I.; BRANCO, I. G.; CONCEIÇÃO, W. A. S.; COUTINHO, M. R. Aproveitamento alternativo da casca do maracujá-amarelo (Passiflora edulis f. var. flavicarpa Deg.) para produção de biscoitos. Revista Ciências Exatas e Naturais, Guarapuava, v. 9, n. 2, p. 279-292, 2007

KOPPER, A. C.; SARAVIA, A. P. K.; RIBANI, R. H.; LORENZI, G. M. A. C. Utilização tecnológica da farinha de bocaiúva na elaboração de biscoitos tipo cookie. Alimentos e Nutrição, Araraquara, v. 20, n. 3, p. 463-469, 2009

LLOBERA, A.; CAÑELLAS, J. Dietary fibre content and antioxidant activity of Manto Negro red grape (Vitis vinifera): pomace and stem. Food Chemistry, London, v. 101, n. 2, p. 658-666, 2007

OSUNA, A. I. L.; AMAVIZCA, O. N. V.; RASCÓN, J. A. V.; ROBLES, A. V.; CASTRO, R. M.; ROMERO, C. R.; WONG, B. R. Elaboration of grape pomace cookies. In: INSTITUTE OF FOOD TECHNOLOGISTS - IFT ANNUAL MEETING, 2004, Las Vegas. Anais Eletrônicos... Las Vegas: IFT, 2004. Disponível em: <http://ift.confex.com/ift/2004/techprogram/paper_26403. htm>. Acesso em: 12 abr. 2012. 
Elaboração e aceitabilidade de biscoitos enriquecidos com aveia e farinha de bagaço de uva

PIOVESANA, A. et al.

ROCKENBACH, I. I. Compostos Fenólicos, Ácidos Graxos e Capacidade Antioxidante do Bagaço da Vinificação de Uvas Tintas (Vitis vinifera e Vitis labrusca). 2008. 112 f. Dissertação (Mestrado em Ciência dos Alimentos)-Universidade Federal de Santa Catarina, Florianópolis, 2008.

ROMERO, R. C.; OSUNA, A. I. L.; SÁNCHEZ, R. M. R.; MORALES, R. C.; LEÓN-MARTINEZ, L.; LEÓN-GÁLVEZ, R. Characterization of cookies made with deseeded grape pomace. Archivos Latinoamericanos de Nutricion, Caracas, v. 54, n. 1 , p. 93-94, 2004.

SANTOS, A. A. O.; SANTOS, A. J. A. O.; SILVA, I. V. C.; LEITE, M. L. C.; SOARES, S. M.; MARCELLINI, P. S. Desenvolvimento de biscoitos de chocolate a partir da incorporação de fécula de mandioca e albedo de laranja. Alimentos e Nutrição, Araraquara, v. 21, n. 3, p. 469-480, 2010.
SANTOS, C. A.; RIBEIRO, R. C.; SILVA, E. V. C.; SILVA, N.; SILVA, B. A. Elaboração de biscoito de farinha de buriti (Mauritia flexuosa L. f) com e sem adição de aveia (Avena sativa L.). Revista Brasileira de Tecnologia Agroindustrial, Ponta Grossa, v. 5, n. 1, p. 262-275, 2011.

SANTUCCI, M. C. C.; ALVIM, I. D.; FARIA, E. V.; SGARBIERI, V. C. Efeito do enriquecimento de biscoitos tipo água e sal com extrato de levedura (Saccharomyces sp.). Ciência e Tecnologia de Alimentos, Campinas, v. 23, n. 3, p. 441-446, 2003. http:// dx.doi.org/10.1590/S0101-20612003000300025

SILVA, M. R.; SILVA, M. S.; MARTINS, K. A.; BORGES, S. Utilização tecnológica dos frutos de jatobá-do-cerrado e de jatobá-da-mata na elaboração de biscoitos fontes de fibra alimentar e isentos de açúcares. Ciência e Tecnologia de Alimentos, Campinas, v. 21, n. 2, p. 176-182, 2001. http://dx.doi. org/10.1590/S0101-20612001000200010 